\title{
The Bologna submillisecond pulsar survey
}

\author{
Nichi D’Amico \\ Bologna Astronomical Observatory, via Ranzani 1, 40127 Bologna, Italy
}

\begin{abstract}
Since the discovery of the original millisecond pulsar, no pulsars with a shorter spin period $(\mathrm{P}<1.56 \mathrm{~ms})$ were found. However, according to the most popular equations of state, the theoretical limiting spin period of a neutron star can be much shorter. On the other hand, most of the large scale searches for millisecond pulsars carried out so far were strongly biased against the detection of ultrashort periodicities. In this paper we describe a new large scale pulsar survey with a minimum detectable period much shorter than previous searches.
\end{abstract}

\section{Introduction}

The discovery of the first millisecond pulsar (MSP) PSR J1939+2134 (Backer et al, 1982), having a rotational period of $\simeq 1.56 \mathrm{~ms}$, rised the challenging question of the limiting spin period of neutron stars (see D'Amico, 1998, and references therein). A sensitive search for ultrafast pulsars requires sophysticated observing equipments, huge data storage devices, and supercomputing facilities, so it is not surprising that the minimum period observed so far is that of the original brigth millisecond pulsar: indeed only periodicities above this value were effectively searched by radioastronomers resulting in the present sample of about 80 objects (in the field and in Globular Clusters) with periods of the order of few milliseconds. The minimum observed period is remarkably close to the limiting spin period of a neutron star predicted by the so called "stiff" equations of state of the ultradense matter. The existence of such a pulsar, and its "clock" stability suggests that this objects must be spinning well above the break-up limit of neutron stars, implying a much lower degree of stiffness of the ultradense matter. Indeed, other realistic and equally qualified equations of state were proposed, resulting in a range of neutron star limiting spin periods. The shortest breakup periods $(\simeq 0.6 \mathrm{~ms})$ are those predicted by the so called "soft" equations of state (see Burderi \& D'Amico, 1997, and reference therein). Possenti et al (1999), showed that Nature could provide evolutionary paths for spinning a significant amount of neutron stars up to such extremely high rotational regime. So, in principle, a search of the submillisecond period range can be used to put constraints on the equation of state of matter at nuclear densities. Triggered by these considerations, we commissioned a new pulsar search experiment at the Northern Cross radiotelescope, near Bologna, Italy. The experiment has enough time resolution to detect these objects, and it is equipped with an online data processing system. It is designed such that very narrow pulses as those of the original millisecond pulsar can be easily detected, and it has a similar sensitivity level for a pulsar spinning near the limiting spin period predicted by the softest 


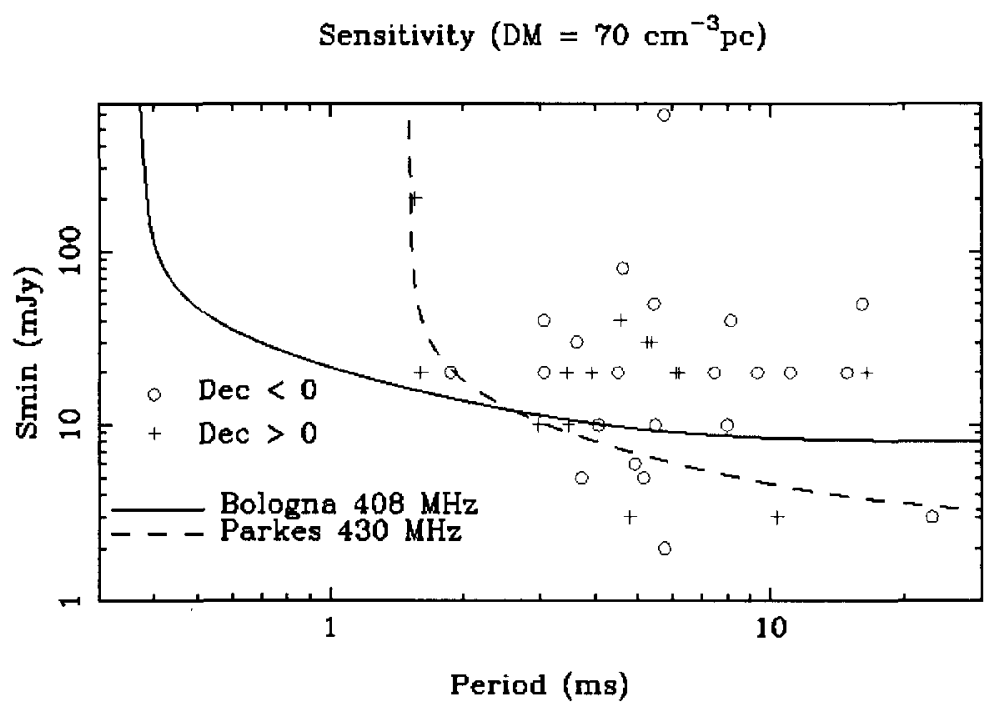

Figure 1. Sensitivity profile of the Bologna survey compared with that of the low frequency Parkes survey (Manchester et al,1996). The positions of the known millisecond pulsars in the Northern and Southern sky are indicated

equation of state $(\simeq 0.6 \mathrm{~ms})$. Substantially, the present experiment represents the first systematic large scale search of the ultrashort period range $(P<1.5 \mathrm{~ms})$.

\section{Sensitivity requirements of the experiment}

The detectability of MSPs is the result of a compromise between several requirements. The minimum detectable pulsar mean flux density is given by:

$$
S_{\min } \times k \frac{S_{S Y S}}{\sqrt{\Delta \nu \Delta t}} \sqrt{\frac{w_{e}}{P-w_{e}}}
$$

where $\mathrm{S}_{S Y S}$ is the system noise equivalent flux density in $\mathrm{Jy}, k$ is a factor that accounts for the adopted detection thereshold and various system losses, (typically $k \simeq 10$ ), $\Delta \nu$ is the observed bandwidth, $\Delta t$ is the integration time, $\mathrm{P}$ is the pulsar period, and $w_{e}$ is the effective pulse width given by

$$
w_{e}=\sqrt{w^{2}+(\delta t)^{2}+\left(\frac{D M}{1.210^{-4}} \frac{\delta \nu}{\nu^{3}}\right)^{2}+\left(\delta t_{\text {scat }}\right)^{2}}
$$

where $w$ is the intrinsic pulse width, $\delta t$ is the time resolution as determined by the sampling time, the post-detection time constant and anti-aliasing filter, $D M$ is the dispersion measure in $\mathrm{cm}^{-3} \mathrm{pc}, \delta \nu$ and $\nu$ are respectively the fre- 
quency resolution and the center observing frequency in $\mathrm{MHz}$, and $\delta t_{\text {scatt }}$ is the broadening of pulses due to multi-path scattering in the interstellar medium.

The scattering term $\delta t_{\text {scat }}$ is roughly proportional to the pulsar distance and scales as $\nu^{-4}$ so, searches at meter wavelengths are usually expected to find mainly nearby, high latitudes millisecond pulsars. The instrument used for the present experiment is the E-W arm of the Northern Cross radiotelescope, near Bologna, Italy, which operates at $408 \mathrm{MHz}$. The experiment parameters are summarized in Table 1, and the corresponding sensitivity is shown in Fig. 1.

\begin{tabular}{|c|c|c|c|c|c|}
\hline \multicolumn{6}{|c|}{ Table 1 - Parameters of the Bologna experiment } \\
\hline $\begin{array}{c}\Delta \nu \\
(\mathrm{MHz})\end{array}$ & $\begin{array}{c}\Delta \nu \\
(\mathrm{MHz})\end{array}$ & $\begin{array}{c}\Delta \mathrm{t} \\
(\mathrm{s})\end{array}$ & $\begin{array}{c}\mathrm{S}_{S Y S} \\
(\mathrm{Jy})\end{array}$ & $k$ & $\begin{array}{c}\delta \mathrm{t} \\
(\mu \mathrm{s})\end{array}$ \\
\hline 408 & 4 & 67 & 45 & 10 & 64 \\
\hline
\end{tabular}

\section{Data acquisition and online processing}

In the Northern Cross pulsar system (D'Amico et al, 1996), the IF signal is split into a filterbank consisting of 128 channels each of width $32 \mathrm{kHz}$. The outputs are detected, low-pass filtered and one-bit digitised. The data acquisition and the hardware set-up are controlled by a PC-style microcomputer using a customized external bus and a digital $\mathrm{I} / \mathrm{O}$ expansion unit. The $\mathrm{PC}$ is used to program the various blocks and log the relevant observation parameters. A special purpose card (D'Amico \& Maccaferri, 1994) is used to manage a data rate up to several megasamples per second, and to keep timing precision. Digital data are transferred to a realtime data processing subsystem using a fast link.

The original feature of the realtime data processing subsystem (Fig. 2) is the dedispersing unit. This is essentially a large bank of 1-bit addressable RAM where the raw digital data of each observed beam position are stored. A special programmable circuitry allows to pick up the the 1-bit samples, sum them with a given dispersion delay, and output the corresponding dedispersed time series to a local bus. Any dedispersed time series transferred on the local bus can be read by any of the four CPUs available on the same bus and searched for periodicities. The most relevant suspect periodicities of each trial DM step are saved into a memory buffer and finally sorted. The most significant suspect periodicities detected on each beam position are saved on a permanent database. An offline program is available to selectively search the database, and classify the suspect by human inspection.

\section{Survey status and results}

So far, we observed about $80 \%$ of the sky region in the declination interval $4^{\circ}$ $<\delta<42^{\circ}$. We have'nt discovered any pulsar with a period shorter or similar to that of the original millisecond pulsar. We have detected 35 known long period pulsars, 5 known millisecond pulsars and 1 new millisecond pulsar PSR $\mathrm{J} 0030+0451$, which was also discovered at Arecibo (Somer et al, these proceedings). This new millisecond pulsar has a period $\mathrm{P}=4.86 \mathrm{~ms}$ and a very low $\mathrm{DM}=4.3 \mathrm{pc}^{-3} \mathrm{pc}$. After the discovery, made early in 1999 , we begun a series of follow-up observations using the Parkes radiotelescope. In particular we made long integrations at $70 \mathrm{~cm}$ in order to measure the scintillation parameters and derive the scintillations velocity (Nigro et al, in preparation). 


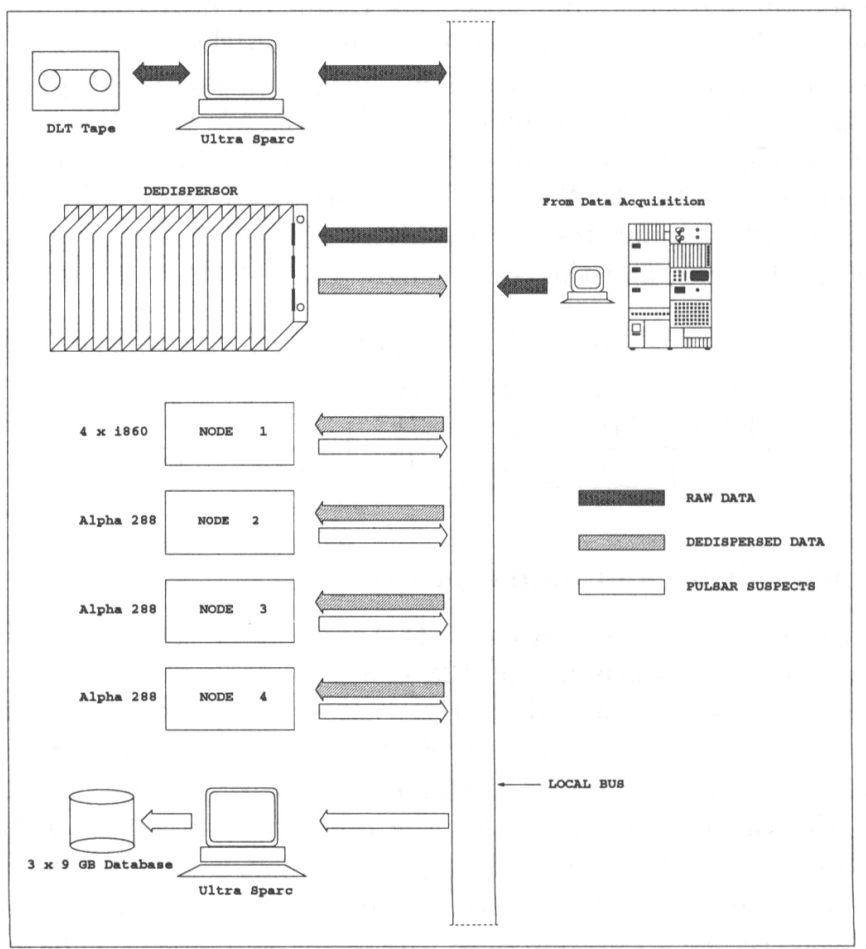

Figure 2. Block diagram of the data processing subsystem

\section{References}

Backer,D.C., Kulkarni, S.R., Heiles, C., Davis, M.M., \& Goss W.M. 1982, Nature, 300,615

Burderi, L., \& D’Amico. N. 1997, ApJ, 490, 343

Manchester, R.N., Lyne, A.G., D'Amico, N., Bailes, M., Johnston, S., Lorimer, D.R., Harrison, P.A., Nicastro, L., \& Bell, J.F. 1996, MNRAS

D'Amico, N., Grueff, G., Montebugnoli, S., Maccaferri A., Cattani, A., Bortolotti, C., Nicastro, L., Fauci, F., Tomassetti, G., Roma, M., Ambrosini, R., \& Rodriguez, E. 1996, ApJS, 106, 611

D’Amico N., \& Maccaferri, A. 1994, Experimental Astronomy, 4, 189

D'Amico N. 1998, in The Many Faces of Neutron Stars. Edited by R. Buccheri, J. van Paradijs, and M. A. Alpar. Dordrecht ; Boston : Kluwer Academic Publishers, 129

Possenti, A., Colpi, M., Geppert, U., Burderi, L., \& D'Amico, N. 1999, these proceedings

Somer, A., Backer, D.C., Zepka, A., Cordes, J.M., Arzoumanian, Z., McLaughlin, M. 1999, these proceedings 